\title{
The Neural Basis of Implicit Attitudes
}

\author{
Damian Stanley, ${ }^{1}$ Elizabeth Phelps, ${ }^{1}$ and Mahzarin Banaji ${ }^{2}$ \\ ${ }^{1}$ New York University and ${ }^{2}$ Harvard University
}

\begin{abstract}
Evidence that human preferences, beliefs, and behavior are influenced by sources that are outside the reach of conscious awareness, control, intention, and selfreflection is incontrovertible. Recent advances in neuroscience have enabled researchers to investigate the neural basis of these implicit attitudes, particularly attitudes involving social groups. From this research, a model with three identified neural components related to the automatic activation and regulation of implicit attitudes is beginning to emerge. The amygdala is implicated in the automatic evaluation of socially relevant stimuli, while the anterior cingulate and dorsolateral prefrontal cortices are involved in the detection and regulation, respectively, of implicit attitudes. Further support for this model comes from the inclusion of these regions in current models concerning the cognitive regulation of emotion and the detection of conflict. The identification of a putative neural substrate for implicit attitudes has had a direct impact on psychological research into their nature and operational characteristics. We discuss how this emerging neural model has influenced current research on implicit attitudes and describe the importance of such models for directing future research.
\end{abstract}

KEYWORDS-implicit attitude; implicit bias; social cognition; social neuroscience; amygdala

Attitudes or preferences are a fundamental component of all living systems. They orient the organism toward or away from people, things, and events in the world. When plants orient toward the sun and flies flee from swatters, their behavior is based on a relatively simple and automatic system of preferences (e.g., tropisms, taxis) without which survival would be compromised. In humans, the levels of preferences and attitudes that guide behavior range from the simple to the highly complex. Attitudes

Address correspondence to Damian Stanley, Department of Psychology, New York University, 6 Washington Place, Rm 865, New York, NY 10003; e-mail: damian.stanley@nyu.edu. enable us to rapidly and efficiently react to simple sensory inputs as well as to multifaceted experiences such as individuals, groups, objects, and events we encounter in our social worlds. Unlike other organisms, humans have the ability to introspectively identify and even change the attitudes they hold. As Max Klinger of $M^{*} A^{*} S^{*} H$ said, "I used to be an atheist, but I gave it up for lent." This ability to examine the contents of our own minds and manipulate them is uniquely human. Its presence can create the illusion that we control more about ourselves and our universe than we actually do, and that we know what our preferences are and why we have them. Research in social psychology has shown that, in addition to our conscious attitudes, we possess automatically triggered attitudes that can influence behavior without our awareness (Fazio, Jackson, Dunton, \& Williams, 1995; Greenwald \& Banaji, 1995). Because these attitudes are "introspectively unidentified (or inaccurately identified)" (Greenwald \& Banaji, 1995, p. 8), they are termed implicit, thereby distinguishing them from explicit attitudes that reflect our conscious thoughts and beliefs. Current models of social cognition posit that human social behavior is the combined result of these two related but distinct sets of attitudes.

\section{IMPLICIT ATTITUDES}

The distinction between implicit and explicit attitudes emerged only in the last 20 years, primarily motivated by findings from cognitive psychology demonstrating the influence of implicit memory and unconscious cognition on behavior (Greenwald \& Banaji, 1995; Bargh \& Chartrand, 1999). While theoretically driven, in practice the distinction between implicit and explicit often ends up being a methodological one. Studies assessing implicit processes use indirect measures over which the participant has little control and of which they may be unaware. Many such measures require participants to respond as quickly as possible, thereby limiting introspection (e.g., Fazio et al., 1995; Nosek, Greenwald, \& Banaji, 2006). Others measure physiological responses, such as skin conductance or indicators of neural activity, to assess participants' automatic reactions to target stimuli or concepts (e.g., Phelps et al., 2000). In contrast, studies 
assessing explicit processes usually require conscious deliberation on the part of the participant and/or rely on self-report.

Indirect measures have been used to assess a wide range of implicit attitudes including, but not limited to, those toward race, gender, sexual orientation, age, religion, and political issues (Greenwald, Poehlman, Uhlmann, \& Banaji, in press; Nosek et al., 2007). While informative about specific attitudes, these data have also provided a wealth of information about the characteristics of implicit attitudes in general. Implicit attitudes predict behavior such as choices, judgments, and nonverbal behaviors toward members of other social groups, and in some cases they can be more informative than explicit attitudes are (Fazio et al., 1995; Greenwald et al., in press). In particular, measures of implicit attitudes outperform measures of explicit attitudes in socially sensitive domains (e.g., stereotyping and prejudice; Green et al., 2007). Implicit attitudes are triggered automatically, often without awareness, in a manner that can depend upon the particular social context (Blair, 2002; Fazio et al., 1995; Greenwald \& Banaji, 1995). In addition, they are pervasive, in that they are found across different demographic groups and topics (Nosek et al., 2007). Implicit attitudes are related to explicit attitudes, but they are distinct in that neither is robustly predictive of the other (Greenwald et al., in press; Nosek et al., 2007).

These qualities have led to interest in implicit attitudes and their influence on behavior across disciplines as diverse as social psychology, neuroscience, law, developmental psychology, and market research (Greenwald et al., in press; Nosek et al., 2007; Phelps \& Banaji, 2005). Accompanying this interest is a desire to better understand the underlying phenomena that give rise to implicit attitudes, as well as to learn how we might regulate their impact on our own behavior. Neuroscientific techniques such as functional magnetic resonance imaging (fMRI) and electroencephalography (EEG) have enabled researchers to begin to elucidate the neural systems involved in the expression and regulation of implicit attitudes. Importantly, these techniques make it possible not only to investigate the neural substrate of implicit attitudes in humans but also to draw comparisons across species, allowing animal models to inform research on implicit attitudes. The merging of neuroscience and psychology has influenced current theories of implicit attitudes and is likely to be an essential component of future discoveries. In particular, current efforts to develop neural models of implicit attitudes are critical for determining their operational characteristics as well as for linking them to other psychological processes (e.g., Phelps \& Banaji, 2005).

\section{THE AMYGDALA AND IMPLICIT ATTITUDES TOWARD RACE}

One significant contribution of neuroscience has been the identification of the amygdala as a brain region involved in the expression of implicit attitudes. The amygdala is a small group of nuclei located bilaterally in the anterior temporal lobe that has been studied extensively with respect to implicit emotional learning and memory, as well as with respect to the automatic effects of emotional stimuli upon cognition and perception (for review, see Phelps \& LeDoux, 2005). Structurally, the amygdala is well situated to combine social and cognitive input and to modulate cognition and automatic aspects of behavior. Input to the amygdala is diverse, including contributions from low-level sensory areas and from brain regions underlying memory and higher cognitive processes. Output from the amygdala is also widespread, ranging from the brain stem and hypothalamus to early sensory cortices and cortical association areas.

A range of studies have demonstrated that, with its diverse projections, the amygdala modulates a range of cognitive functions, such as memory and attention, as well as autonomic responses, in the presence of emotionally salient stimuli (Phelps \& LeDoux, 2005). In particular, the amygdala has been shown to be critical for fear learning-that is, the implicit, physiological expression of emotional responses to neutral stimuli that have been paired with an aversive stimulus. Importantly, the amygdala is also recruited when fear learning is induced indirectly, either through observation or instruction (Phelps \& Banaji, 2005; Phelps \& LeDoux, 2005), indicating that it is sensitive to the types of social cues imperative in the formation of implicit attitudes. The amygdala's role in evaluative aspects of social interactions is further supported by lesion and functional imaging work in monkeys (Adolphs, 2003) and humans (Phelps \& LeDoux, 2005).

The amygdala was first linked to implicit attitudes in a study by Phelps et al. (2000). The authors used fMRI to measure bloodoxygen-level dependent (BOLD) responses in White American participants while they viewed faces of unfamiliar Black and White males. In addition, they collected behavioral measures assessing the participants' implicit and explicit race-related attitudes. The magnitude of the amygdala BOLD response when participants viewed Black (compared to White) faces was significantly correlated with measures of individuals' implicit, but not explicit, race-related behavioral attitudes (see Fig. la). These data suggested that the amygdala plays a role in the evaluation of other social groups and that this role reflects individual experience.

To further examine how experience might modulate amygdala activity, Phelps et al. (2000) repeated the experiment using pictures of famous, positively regarded Black and White men rather than strangers. In this second experiment, the correlation between BOLD responses in the amygdala and participants' implicit race-related attitudes was eliminated. Another study, by Hart et al. (2000), also used fMRI to investigate amygdala activity while both Black and White observers viewed unfamiliar Black and White faces. They found that the BOLD response in the amygdala, which was initially elevated to unfamiliar faces of both races, habituated over time to same-race faces but not to other-race faces. One interpretation of these data, given the 
(a)
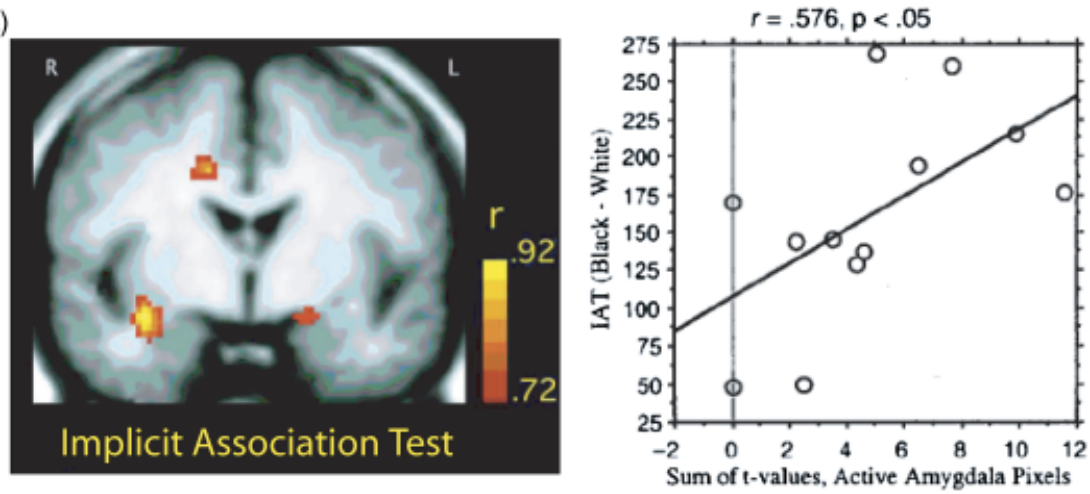

(b)
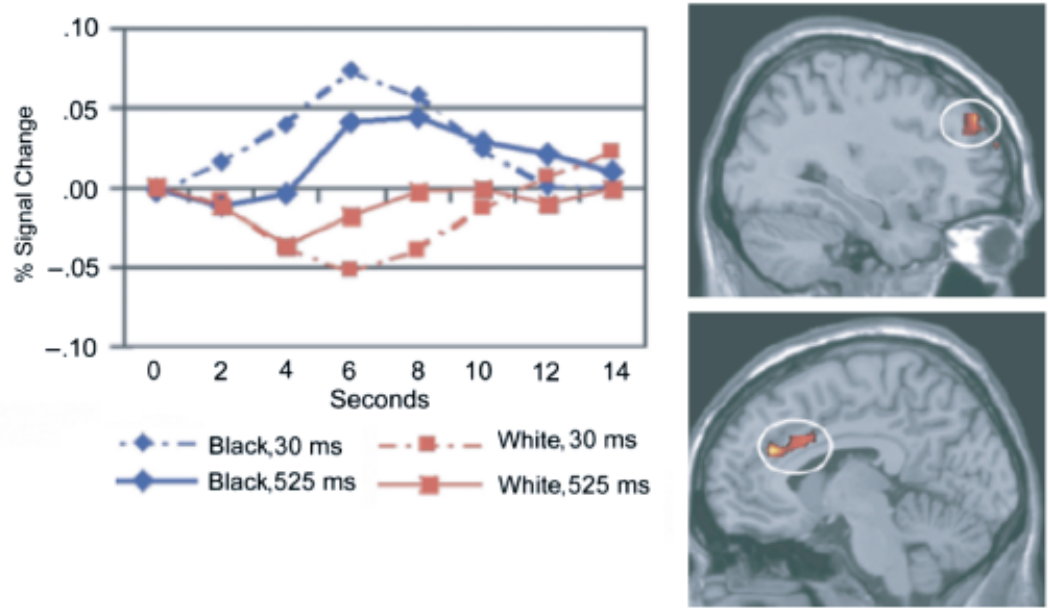

Fig. 1. Amygdala responses to other-race faces correlated with measures of implicit attitudes (panel a) and neural regions implicated in the automatic and controlled processing of other-race faces (panel $b$ ). The coronal slice (a, left) shows activation in the amygdala for which the magnitude of the response to Black (contrasted with White) faces is correlated with White participants' implicit attitudes toward Blacks as measured by the Implicit Association Test (IAT; see Nosek, Greenwald, \& Banaji, 2006 for details). The graph (a, right) indicates the correlation between individual participants' amygdala response ( $x$-axis) and their implicit attitudes toward Blacks as measured by the IAT ( $y$-axis). The lower graph (b, left) shows timecourses of the response in the amygdala to supraliminally ( 525 milliseconds) and subliminally ( 30 milliseconds) presented Black and White faces. Note that the magnitude of the response for subliminally presented Black (compared to White) faces is larger than for those presented supraliminally, implicating the amygdala in the automatic evaluation of other-race faces. The panels at the right show activation to supraliminally presented Black (contrasted with White) faces in the dorsolateral prefrontal cortex (dIPFC, top) and anterior cingulate cortex (ACC, bottom), implicating these regions in the controlled processing of other-race faces. Panel a adapted from "Performance on Indirect Measures of Race Evaluation Predicts Amygdala Activation," by E.A. Phelps, K.J. O'Connor, W.A. Cunningham, E.S. Funayama, J.C. Gatenby, J.C. Gore, \& M.R. Banaji, 2000, Journal of Cognitive Neuroscience, 12, pp. 729-738; copyright 2000 by the Massachusetts Institute of Technology; adapted with permission. Panel b adapted from Cunningham et al. (2004).

amygdala's role in emotional responses to threat stimuli, is that alarm signals from the amygdala attenuate more rapidly for same-race than for other-race strangers.

A defining characteristic of implicit attitudes is that they are expressed automatically, often without the knowledge of the person expressing them (Greenwald \& Banaji, 1995). Previous findings in the emotion literature have demonstrated that conscious perception is not required to generate the amygdala response to fearful faces and other stimuli that may signify potential threat (Phelps \& LeDoux, 2005). If the amygdala is involved in the activation of race-related implicit attitudes, then conscious perception of an implicit-attitude-inducing stimulus should not be a condition for its recruitment.

Cunningham et al. (2004) tested this hypothesis by collecting fMRI data while White Americans viewed pictures of Black and White unfamiliar faces that were presented either subliminally (i.e., not consciously seen by the participant) or supraliminally (i.e., consciously seen). They found that enhanced BOLD responses in the amygdala to Black versus White faces were significantly stronger in the subliminal condition than they were in the supraliminal condition (Fig. 1b). This finding is interesting for two reasons. First, it demonstrates that the amygdala is 
automatically recruited when viewing other-race faces, replicating and extending the findings of Phelps et al. (2000). Second, the evidence for a stronger subcortical signal when the stimuli are presented below the threshold for conscious perception suggests that an automatic amygdala response is suppressed when participants consciously perceive other-race faces. Cunningham et al. (2004) suggested that this suppression may be the result of regulatory mechanisms that control the expression of race-related implicit attitudes so that behavior reflects our explicit attitudes. Indeed, they found that amygdala activity elicited by the supraliminal presentation of faces reflected the combination of participants' implicit and explicit attitudes. It should be noted, however, that although the amygdala seems to be automatically recruited during the activation of race-related implicit attitudes, it may not be critical for all aspects of the behavioral response. Phelps, Cannaistraci, \& Cunningham (2003) found intact behavioral evidence for implicit attitudes in a patient with bilateral amygdala damage, showing that the amygdala may not be directly involved in mediating all effects of race-related implicit attitudes on behavior.

\section{NEURAL SYSTEMS INVOLVED IN THE REGULATION OF RACE-RELATED IMPLICIT ATTITUDES}

The finding that the amygdala's response is suppressed when participants are aware of the other-race faces (Cunningham et al., 2004) suggests that other regions of the brain may be detecting the presence of attitude-inducing stimuli and modulating the amygdala's automatic response. To investigate this possibility, Cunningham et al. (2004) calculated an index of amygdala modulation for the supraliminal compared to the subliminal condition. They found that activity in the dorsolateral prefrontal cortex (dlPFC) and the anterior cingulate cortex (ACC; Fig. 1b) was correlated with attenuation of the amygdala response to supraliminal Black faces in White subjects. They interpreted these findings as providing evidence that the dIPFC and ACC were involved in the regulation of "spontaneously activated negative attitudes" (p. 811). This regulatory role for the dlPFC is also supported by Knutson et al. (2007), who found that the dIPFC was engaged when participants were required to categorize stimuli in a manner counter to their race- and genderrelated implicit attitudes.

A study by Richeson et al. (2003) further elucidates the potential roles of the AIPFC and ACC in regulating the activation of race-related implicit attitudes. First, they assessed how White participants' executive-control processes were impaired following an interracial interaction. From this measure, they inferred the extent to which participants utilized executive control to combat the activation of anti-Black implicit attitudes during the interracial interaction. In a separate session, they collected fMRI data while the same participants viewed Black and White faces. They found that activity in both the ACC and the dIPFC to other-race faces was sensitive to the extent to which participants utilized executive control during the interracial interaction. Additionally, they found that only activity in the dIPFC, and not the ACC, remained a significant predictor of the utilization of executive control when individuals' race-related implicit attitudes were taken into account. Based on these findings and prior studies, the authors suggest that the dIPFC is involved in engaging cognitive control during interracial interactions and that the ACC is involved in detecting the need for engaging cognitive control. This role for the ACC is further supported by EEG studies demonstrating that neural activity associated with the ACC reflects the detection of conflict between implicit attitudes and explicit goals (see Amodio, Harmon-Jones, \& Devine, 2007).

Together, these studies suggest how the brain might regulate the automatic activation of implicit attitudes using built-in mechanisms for cognitive control. Of great interest are behavioral studies that demonstrate variation in implicit attitudes as a function of social context and the goals and motivations of the participant (Blair, 2002). The context of a situation may modulate the activation of implicit attitudes without engaging regulatory mechanisms. While this has yet to be directly addressed, there is evidence that task goals can modulate the amygdala response to Black and White faces. Wheeler and Fiske (2005) found a heightened amygdala response to Black (compared to White) faces when participants were directed to focus on social category (i.e., age); such a response vanished when attention was directed away from social category and toward the individual person. Likewise, Lieberman, Hariri, Jarcho, Eisenberger, \& Bookheimer (2005) found race-related activity in the amygdala when participants performed a perceptual-encoding task but not when they performed a verbal-encoding task; the latter resulted instead in race-related activation of the ventrolateral prefrontal cortex. These studies demonstrate that social-interaction goals can affect which neural systems are activated and engaged in a given situation.

\section{TOWARD A NEURAL MODEL FOR THE ACTIVATION AND REGULATION OF RACE-RELATED IMPLICIT ATTITUDES}

The findings reviewed above are consistent with a neural model for race-related implicit attitudes with at least 3 components (Fig. 2). The automatic activation of race-related implicit attitudes is correlated with the amygdala BOLD response, implicating the amygdala in the automatic evaluation of socially relevant stimuli. Given the complexity of the human social environment, relying only or even primarily on automatic preferences to drive behavior is not an optimal system for the survival of the organism. Successful operation in the social world involves interactions between individuals who come from a variety of different social groups. Perhaps for this reason, human evolution has also granted the capacity for modulating the impact of 


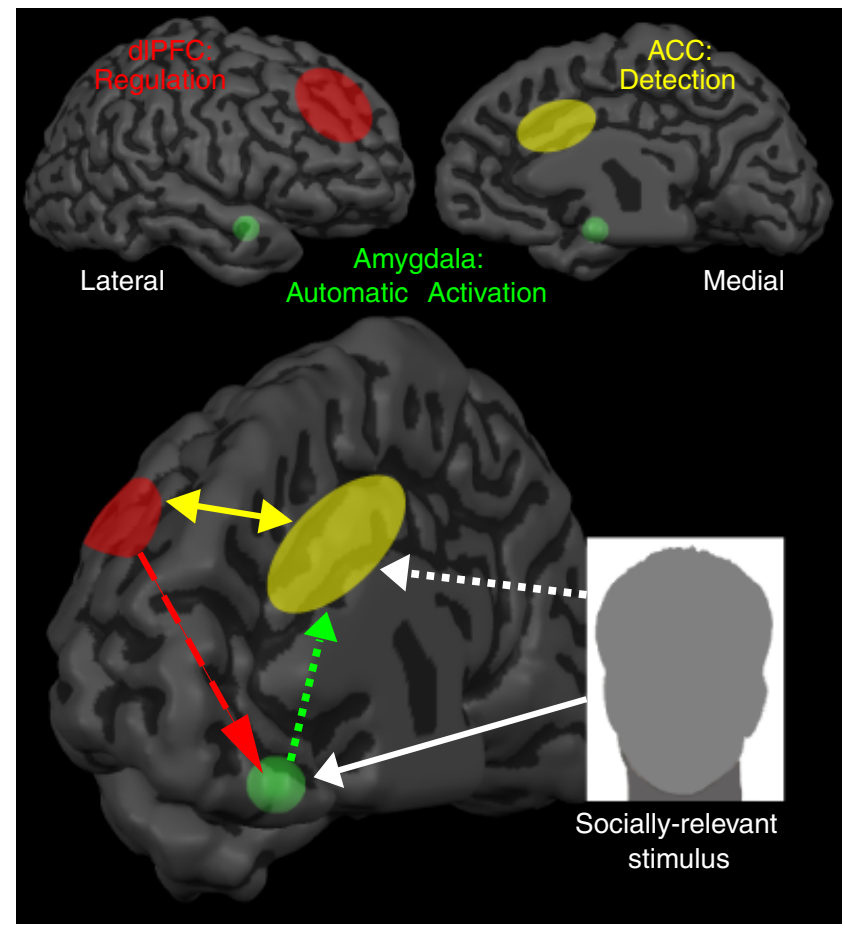

Fig. 2. A model for the neural basis of implicit attitudes. The evidence reviewed in this article suggests at least 3 components: The amygdala is implicated in the automatic evaluation of socially relevant stimuli while the anterior cingulate cortex (ACC) and the dorsolateral prefrontal cortex (dIPFC) are implicated in the detection of such stimuli and the regulation of the amygdala's response, respectively. There are many open questions. For example, how does the dIPFC exert its influence over the amygdala given that there is little evidence of direct connectivity between the two structures (dashed red arrow; one possibility is via the ventromedial prefrontal cortex — see Phelps and LeDoux, 2006)? Another is whether the ACC detects the presence of a social stimulus itself (dotted white arrow) or is, instead, sensitive to the initiation of the automatic amygdala response (dotted green arrow).

implicit attitudes on our behavior. The dIPFC may play this role, regulating amygdala responses when it is "aware" that an implicit attitude has been triggered. It should be noted that there is little evidence of direct anatomical connection between the dIPFC and the amygdala, raising the question of how this modulatory role for the dIPFC might be mediated. One possibility is that the dIPFC exerts its effects indirectly via the ventral and/or medial prefrontal cortex (Phelps \& LeDoux, 2005). Finally, the data suggest that the ACC may subserve the detection of conflict between implicit and explicit attitudes, signaling the need for the activation of control mechanisms. Recent findings examining the cognitive control of emotion, as well as other types of cognitive control and conflict monitoring, have implicated similar regions of the prefrontal cortex and the ACC, providing further support for the validity of the current model (Amodio et al., 2007; Phelps \& LeDoux, 2005, see also, Ochsner \& Gross, 2008 , this issue).

Although still in the early stages of development, this emerging neural model has already had a clear impact on models of implicit attitudes. In one respect, evidence for a common neural substrate for systems of emotion, cognitive regulation, and implicit attitudes has been used to bolster psychological claims about the nature of implicit attitudes. The linking of racerelated implicit attitudes to amygdala function has strengthened claims of those attitudes' unconscious and automatic nature and bolstered arguments that they represent an evolutionarily conserved evaluative process. Similarly, the identification of distinct neural systems for the expression and regulation of implicit attitudes, and the established involvement of those systems in implicit and explicit processes (Amodio et al., 2007; Phelps \& Banaji, 2005), is further evidence for the distinction between implicit and explicit attitudes. However, the main strength of this model is not its use to validate existing theories; rather, it is that researchers can now draw from an extensive neuroscientific literature to make novel predictions about the operational characteristics of implicit attitudes.

A recent study by Olsson, Ebert, Banaji, \& Phelps (2005) did just this, capitalizing on findings that implicit attitudes and fear learning have a common neural substrate. From the fear-learning literature, it is known that learned associations between biologically "prepared" stimuli (i.e., stimuli representing natural threats such as snakes and spiders) and aversive stimuli (e.g., a shock) persist longer than learned associations between neutral and aversive stimuli (see Phelps \& LeDoux, 2005). Olsson et al. (2005) investigated whether other-race faces were like prepared stimuli. They showed that the association of other-race faces with an aversive stimulus persisted longer than did such an association with same-race faces, for both Black and White American participants. Interestingly, this effect was reduced in participants who had been involved in an interracial relationship, indicating that personal experience can modulate the strength of association between other-race faces and aversive stimuli.

Identifying a neural substrate for implicit attitudes has also enabled researchers to investigate the subprocesses involved in their regulation and expression. Historically, the methods of experimental psychology have been constrained by behavioral measures (e.g., self-report, response latencies) and physiological measures (e.g., skin conductance, pupil dilation) that can only reveal the end result of neural computations that give rise to a behavior. Neuroscientific methods can extend this work by uncovering the distinct contributions of multiple systems to a given psychological process. A clear example of this is the identification of distinct roles for the ACC and the dIPFC in the detection of conflict between implicit and explicit systems and in cognitive regulation, respectively (Amodio et al., 2007; Cunningham et al., 2004; Richeson et al., 2003). These findings have served to refine models accounting for the regulation of racebased implicit attitudes (see Amodio et al., 2007 for review). Most early models of implicit-attitude regulation posited that control mechanisms required conscious reflection to engage. However, the evidence now suggests that the detection of conflict between implicit and explicit beliefs, as well as some aspects of 
their regulation, is an automatic process that is not dependent on the allocation of cognitive resources and may be outside the perceiver's conscious goals in the immediate moment. The value of describing subprocesses such as these that contribute to the expression of implicit attitudes is readily apparent. Future work should focus on further refining our understanding of the neural systems that act in concert to produce implicit-attitude-related behaviors, as well as the sensitivity of these systems to situational factors.

\section{CONCLUSION}

The discovery that implicit attitudes can automatically and unconsciously influence behavior is of great interest not only to behavioral scientists but also to those concerned with the impact of such attitudes on social well-being in general. Recent efforts to elucidate the neural basis of implicit attitudes have identified a likely subset of brain regions, including the amygdala, dIPFC, and ACC, whose activity reflects the automatic expression, detection, and cognitive regulation of race-related implicit attitudes. This model has already shaped research programs on implicit attitudes, serving to both validate and refine psychological models. However, it is still in its infancy, and therefore it is safe to say that its true potential remains untapped. Future work should focus on identifying other neural systems that contribute to the expression of implicit attitudes involving other social groups, as well as systematically characterizing the functional properties of those systems already identified.

fMRI is a useful tool for examining these questions; however, it is primarily correlational and therefore poorly suited for establishing causality. Researchers should employ other techniques, such as transcranial magnetic stimulation, lesion, and neurophysiological studies, as well as the construction of animal models, to demonstrate causality and to identify the processes necessary for the expression and regulation of implicit attitudes. The fusion of neurobiological and psychological findings into a single model of how implicit attitudes are represented, expressed, and regulated is critical for understanding how these attitudes affect, and are affected by, our social interactions and environment. Through this understanding, the construction of our social environment can be informed and adapted to take into account implicit attitudes that potentially run counter to our conscious objectives and beliefs.

\section{Recommended Reading}

Mao, G.R., \& Olson, J.M. (Eds.). (2000). Why we evaluate: Functions of attitudes. Mahwah, NJ: Erlbaum. A book concerning the role attitudes play in various psychological phenomena.

Nosek, B.A., Greenwald, A.G., \& Banaji, M.R. (2006). (See References). A methodological and conceptual review of the Implicit Association Test (IAT).
Phelps, E.A., \& Banaji, M.R. (2005). (See References). A review of the neuroscientific literature detailing the role of the amygdala in emotion processing.

Phelps, E.A., \& LeDoux, J.E. (2005). (See References). A chapter detailing the relationship between animal models of emotion processing and recent work on the neural basis of human attitudes.

Wittenbrink, B., \& Schwartz, N. (Eds.). (2007). Implicit measures of attitudes. New York: Guilford. A book dedicated to detailing and critiquing the procedures used to obtain implicit measures of attitudes.

Acknowledgments-We would like to thank the Third Millennium Foundation and the Mind Science Foundation for funding this work.

\section{REFERENCES}

Adolphs, R. (2003). Cognitive neuroscience of human social behaviour. Nature Reviews Neuroscience, 4, 165-178.

Amodio, D.M., Harmon-Jones, E., \& Devine, P.G. (2007). Mechanisms for the regulation of intergroup responses: Insights from a social neuroscience approach. In E. Harmon-Jones \& P. Winkielman (Eds.), Fundamentals of social neuroscience (pp. 353-375). New York: Guilford.

Bargh, J.A., \& Chartrand, T.L. (1999). The unbearable automaticity of being. American Psychologist, 54, 462-479.

Blair, I.V. (2002). The malleability of automatic stereotypes and prejudice. Personality \& Social Psychology Review, 6, 242-261.

Cunningham, W.A., Johnson, M.K., Raye, C.L., Chris Gatenby, J., Gore, J.C., \& Banaji, M.R. (2004). Separable neural components in the processing of Black and White faces. Psychological Science, $15,806-813$.

Fazio, R.H., Jackson, J.R., Dunton, B.C., \& Williams, C.J. (1995). Variability in automatic activation as an unobtrusive measure of racial attitudes: A bona fide pipeline? Journal of Personality and Social Psychology, 69, 1013-1027.

Green, A., Carney, D., Pallin, D., Ngo, L., Raymond, K., Iezzoni, L., \& Banaji, M. (2007). Implicit bias among physicians and its prediction of thrombolysis decisions for Black and White patients. Journal of General Internal Medicine, 22, 1231-1238.

Greenwald, A.G., \& Banaji, M.R. (1995). Implicit social cognition: Attitudes, self-esteem, and stereotypes. Psychological Review, $102,4-27$.

Greenwald, A.G., Poehlman, A., Uhlmann, E., \& Banaji, M.R. (in press). Understanding and interpreting the Implicit Association Test: III. Meta-analysis of predictive validity. Journal of Personality and Social Psychology.

Hart, A.J., Whalen, P.J., Shin, L.M., McInerney, S.C., Fischer, H., \& Rauch, S.L. (2000). Differential response in the human amygdala to racial outgroup vs ingroup face stimuli. Neuroreport, 11, 23512355.

Knutson, K.M., Mah, L., Manly, C.F., \& Grafman, J. (2007). Neural correlates of automatic beliefs about gender and race. Human Brain Mapping, 28, 915-930.

Lieberman, M.D., Hariri, A., Jarcho, J.M., Eisenberger, N.I., \& Bookheimer, S.Y. (2005). An fMRI investigation of race-related amygdala activity in African-American and Caucasian-American individuals. Nature Neuroscience, 8, 720-722. 
Nosek, B.A., Greenwald, A.G., \& Banaji, M.R. (2006). The Implicit Association Test at age 7: A methodological and conceptual review. In J.A. Bargh (Ed.), Social psychology and the unconscious: The automaticity of higher mental processes (pp. 265-292. Psychology Press.

Nosek, B.A., Smyth, F.L., Hansen, J.J., Devos, T., Lindner, N.M., Ranganath, K.A., et al. (2007). Pervasiveness and correlates of implicit attitudes and stereotypes. European Review of Social Psychology, 18, 36-88.

Ochsner, K.N., \& Gross, J.J. (2008). Cognitive emotion regulation: Insights from social cognitive and affective neuroscience. Current Directions in Psychological Science, 17, 153-158.

Olsson, A., Ebert, J.P., Banaji, M.R., \& Phelps, E.A. (2005). The role of social groups in the persistence of learned fear. Science, 309, 785-787.

Phelps, E.A., \& Banaji, M.R. (2005). Animal models of human attitudes: Integrations across behavioral, cognitive and social neuroscience. In J.T. Cacioppo, P.S. Visser, \& C.L. Pickett (Eds.), Social neuroscience: People thinking about people. Cambridge, MA: MIT Press.
Phelps, E.A., Cannaistraci, C.J., \& Cunningham, W.A. (2003). Intact performance on an indirect measure of race bias following amygdala damage. Neuropsychologia, 41, 203-208.

Phelps, E.A., \& LeDoux, J.E. (2005). Contributions of the amygdala to emotion processing: From animal models to human behavior. Neuron, 48, 175-187.

Phelps, E.A., O'Connor, K.J., Cunningham, W.A., Funayama, E.S., Gatenby, J.C., Gore, J.C., \& Banaji, M.R. (2000). Performance on indirect measures of race evaluation predicts amygdala activation. Journal of Cognitive Neuroscience, 12, 729-738.

Richeson, J.A., Baird, A.A., Gordon, H.L., Heatherton, T.F., Wyland, C.L., Trawalter, S., \& Shelton, J.N. (2003). An fMRI investigation of the impact of interracial contact on executive function. Nature Neuroscience, 6, 1323-1328.

Wheeler, M.E., \& Fiske, S.T. (2005). Controlling racial prejudice: Social-cognitive goals affect amygdala and stereotype activation. Psychological Science, 16, 56-63. 